\title{
Diseño de un instrumento para identificar las prioridades competitivas del sector maquilador en San Pedro Sula
}

\author{
Jared Roberto Ocampo Martínez ${ }^{1}$ \\ UNITEC, San Pedro Sula, Honduras.
}

(Recibido: Diciembre, 2014 y Aceptado para publicación: Noviembre, 2015)

\begin{abstract}
Resumen:
La globalización ha creado la necesidad que las industrias maquiladoras replanteen sus estrategias y procedimientos para volverse más competitivas y poder de esta forma mantenerse en el mercado. Para poder lograr esta competitividad, las empresas deben establecer estrategias de manufactura que estén enfocadas en satisfacer las necesidades específicas de los clientes. Las necesidades de los clientes pueden modelarse en base al énfasis que estos colocan a ciertas prioridades que compiten entre sí. Una empresa que logra entender bien cuáles son estas prioridades; y luego trabaja para que su estrategia de manufactura responda a estas necesidades logrará posicionarse positivamente sobre sobre su competencia. Este artículo propone un instrumento y metodología que permita identificar las prioridades competitivas del mercado maquilador, así como un indicador que determine que tan bien alineada esta una empresa con dichas prioridades meta. Para hacerlo se ha hecho un extenso análisis de la literatura y se han realizado entrevistas con expertos para probar la validez de constructo del instrumento diseñado.
\end{abstract}

Palabras Claves: Prioridades competitivas, Industria maquiladora, Ensamblaje de exportación, Métodos de investigación empírica.

\begin{abstract}
:
Globalization has created the need for the manufacturing industry to rethink their strategies and processes to become more competitive and thus able to remain in the market. To achieve this competitiveness, companies must establish manufacturing strategies that are focused on meeting the specific need of customers. Customer needs can be modeled based on the emphasis they place on certain competing priorities. A company that understands the priorities and develops a strategy that responds to the needs of the industry, will be able to position itself positively over its competition. The following paper proposes an instrument and methodology to identify the competitive guidelines of the manufacturing market as well as indicators to determine how well aligned is a company with target priorities. To do it, and extensive analysis of the literature and interviews with experts was made to validate and develop the instruments designed.
\end{abstract}

Keywords: Competitive priorities, Maquiladora industry, Assembly for export, Empirical research methods.

\section{Introducción}

La globalización de la economía ha abierto los mercados, de forma que las empresas tienen libertad de ofrecer bienes y servicios o de adquirirlos de cualquier oferente a nivel mundial. En un esfuerzo para mejorar sus niveles de competitividad, muchas compañías han buscado abastecerse de lugares en el mundo que ofrezcan la mejor mezcla entre el valor y el costo posible, fabricando sus productos en países con una mano de obra barata y vendiendo en mercados en donde puedan obtener el precio de venta más alto posible (Dornier et al. 1998; Sarache et al. 2011). Esta necesidad ha dado lugar a la creación de la industria del ensamblaje orientada a la exportación, en la cual una corporación multinacional establece fábricas industriales internacionales en países en vías de

\footnotetext{
${ }^{1}$ Autor para correspondencia. Email: jared.ocampo@unitec.edu
} 
desarrollo para producir productos y servicios a precios más cómodos que son luego comercializados en naciones desarrolladas (Seguino y Grown, 2006).

Esta industria de ensamblaje orientada a la exportación es conocida en Latinoamérica como "industria de la maquila" y las plantas en donde su actividad productiva se desarrolla como plantas maquiladoras. La llegada de las maquilas a lugares como México, Centroamérica y el Caribe ha impulsado la actividad manufacturera de estos países de forma significativa, generando empleos directos para miles de personas e ingresos para las comunidades beneficiadas (Alonso, et al. 2002; Interiano, 2004; CEPAL, 2005). Las empresas maquiladoras forman parte del tejido industrial productivo de Honduras, principalmente en el sector de San Pedro Sula, en donde son miles las personas cuyo empleo depende de su éxito.

De acuerdo a CEPAL (2004), existe gran preocupación por pérdidas de competitividad experimentadas en la llamada cadena del "hilo-textil-confección", producto de cambios en la organización industrial de la industria maquiladora de exportación y de las estrategias que estas empresas están usando. Uno de los desafíos actuales más importantes es la introducción del esquema denominado "paquete completo" (full packaging). Esto significa que la empresa maquiladora negocia con su cliente un contrato en donde se hace responsable de adquirir todos los insumos, accesorios y maquinarias a cambio de establecer el cumplimiento de estándares, tiempos de entrega y calidad de los productos finales que entregará. Este esquema aumenta la capacidad de mejorar los márgenes de ganancia del subcontratista por causa de los ahorros que pude obtener al buscar eficiencias en diferentes procesos, pero también aumenta el riesgo de no cumplir con los requisitos pactados. Esto requiere de una mejora notable en la competitividad de la empresa.

Para lograr el aumento en la competitividad y desempeño de una empresa es de vital importancia tomar decisiones y realizar acciones dirigidas a mejorar las capacidades de manufactura de dicha empresa. Para ayudar en esta tarea, es necesario diseñar estrategias que asistan a los gerentes en la toma de decisiones operacionales que afecten la estrategia de manufactura de la empresa y mejoren así su desempeño y competitividad.

\section{Problema de investigación}

La presión enfrentada por las empresas producto de la globalización de los mercados y de las operaciones de manufactura también es aplicable a la industria maquiladora. La entrada de Asia y particularmente China al mercado oferente de producción de ensamble, junto con la desaceleración de la economía mundial ha presentado serios problemas a las empresas maquiladoras hondureñas. Esto significa que las maquiladoras hondureñas están obligadas a replantear sus estrategias, procesos y procedimientos para ser más competitivas y mantenerse en el mercado.

De acuerdo al modelo presentado por Porter (1980), dicha competitividad se desarrolla estableciendo una clara estrategia de empresa que busque ya sea el liderazgo por costos (bajo costo), o una diferenciación por causa de beneficios o características únicas relativas al resto de las empresas. La estrategia que la empresa decide seguir (denominado Plan Estratégico) es el proceso "mediante el cual una organización define su negocio, la visión de largo plazo y las estrategias para alcanzarla, con base al análisis de sus fortalezas, debilidades, oportunidades y amenazas" (Serna, 2007). Muchas compañías han entendido que para mejorar su desempeño en el mercado necesitan una estrategia de manufactura 
con contenidos que estén alineados con los requerimientos de dicho mercado (VivaresVergara, et al. 2014). Para hacer esto primero es necesario entender que es lo que el mercado está demandando para de esta forma poder dar respuesta a esas necesidades.

Según Cozzarin (2006), uno de los elementos fundamentales para lograr desarrollar un sistema productivo que logre una ventaja competitiva duradera, es la definición del conjunto de prioridades competitivas que debe seguir una empresa. Estas prioridades competitivas han ido cambiando con el paso del tiempo y varían en importancia dependiendo de la región y del rubro en el que se estudia (Leong, et al. 1990; Avella, et al. 2001; Yang, et al. 2010). Para que la industria maquiladora pueda desarrollar estrategias que le permitan ser competitivos necesita saber cuales son las prioridades competitivas (factores) y las dimensiones (componentes) que el mercado demanda de ellos. Sin embargo, a pesar de que se han realizado muchos estudios sobre la temática de prioridades competitivas de diferentes industrias, no se ha encontrado en la literatura uno que identifique las prioridades competitivas que debería de tener la industria maquiladora en San Pedro Sula desde la perspectiva de sus clientes.

\subsection{Objetivos de investigación}

El problema de investigación antes presentado conduce a hacerse la siguiente pregunta: ¿Cómo pueden estimarse los requerimientos de mercado en cuanto a las prioridades competitivas que debería tener una empresa maquiladora? Por lo tanto, para dar respuesta a esta pregunta, el objetivo de este proyecto de investigación es el siguiente:

Crear un instrumento y metodología que permita obtener un modelo de referencia de los requisitos de mercado de la industria maquiladora (prioridades competitivas de mercado y sus factores) en San Pedro Sula.

\section{Revisión bibliográfica}

La siguiente revisión de bibliografía en los temas relacionados con estrategia de manufactura, modelos teóricos de prioridades competitivas, ponderaciones de factores y componentes, y validación de instrumentos, se hace para ayudar a guiar la metodología que se seguirá para cumplir los objetivos de este proyecto.

\subsection{Estrategia de manufactura}

Se ha realizado mucha investigación sobre la relación existente entre la estrategia de manufactura que sigue una empresa y el desempeño de la misma (Ward, et al. 1998; Buttler y Leong, 2000; Dangayach y Deshmukn, 2006; Yang, et al. 2010). El concepto de estrategia de manufactura fue definido por Skinner (1969) como la explotación de ciertas propiedades de la función de manufactura para lograr ventajas competitivas. Hallgren (2007) apuntó que las dos propiedades más importantes de dicha estrategia son las decisiones tomadas que determinan las capacidades del sistema y la existencia de objetivos de manufactura específicos. Estos objetivos deben de estar alineados con los requerimientos del mercado para lograr que la empresa sea más competitiva. Leong, et al. (1990) da a estos elementos los nombres de prioridades competitivas y categorías de decisión. Hallgren (2007) explica que a través de estos dos elementos se puede entender la capacidad de manufactura de una planta, que es la conexión existente entre el contenido de la estrategia de manufactura y el desempeño del sistema de manufactura (Figura 1). 
Debido a que el término "competencia" puede entenderse como el grado de especialización o excelencia que se tiene en cierta área en comparación con la competencia, se puede decir que la competencia puede medirse por la capacidad que se tiene en cierta área funcional (De Carolis, 2003). Por lo tanto, la capacidad de manufactura es la fortaleza o habilidad que una unidad de negocio tiene para lograr cierto desempeño esperado el cual es medido usando métricas de desempeño operacionales (Ferdows y De Meyer, 1990; Größler y Grübner, 2006).

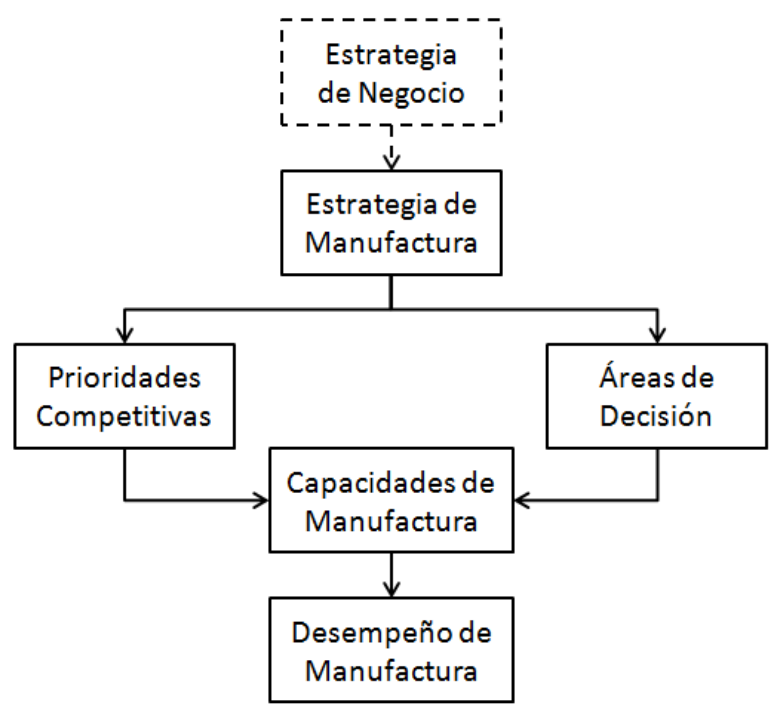

Figura1. Modelo del contenido de la estrategia de manufactura

Fuente: Elaboración propia a partir de Leong, et al. 1990; Größler y Grübner, 2006 y Hallgren, 2007

De la revisión de varios autores se obtiene que las cuatro prioridades competitivas de manufactura fundamentales son costo o eficiencia, flexibilidad, calidad y tiempo de entrega (Skinner, 1969; Hayes y Wheelwright, 1984; Fine y Hax, 1985; Avella, et al. 2001, Greasley, 2009). Sin embargo, otros autores agregan también la innovación (Leong, et al. 1990; Corbett y Van Wassenhove, 1993; Tan, et al. 2007), el servicio al cliente (Davis, et al. 2001; Da Silveira y Slack, 2001), la protección del medio ambiente (Álvarez, et al. 2001; Avella, et al. 2011) y la experiencia o "know-how" (Phusavat y Kanchana, 2007; Nambirajan y Prabhu, 2011). La Tabla 1 presenta una descripción de cada una de estas prioridades competitivas.

Fine y Hax (1985) mencionan que es imposible sobresalir en todas estas prioridades de manera simultánea y por eso es necesario realizar un equilibrio o trade-off entre ellas. Para esto se sugiere ordenar las prioridades competitivas por su peso relativo, de acuerdo a su orden de importancia para el mercado o la empresa. Usando este orden jerárquico es posible definir cuáles son las tareas que la unidad de manufactura debe de ejecutar bien para alcanzar los requisitos de mercado que le permitirán a la empresa ser competitiva (Porter, 1998; Martínez y Bititci, 2006), o en donde debe de enfocar más recursos (Kathuria, 2000; Boyer y Lewis, 2002). Tener congruencia entre las prioridades competitivas que se persiguen y las decisiones de manufactura que se toman para lograrlo (capacidades de manufactura de la empresa), permitirá un rendimiento empresarial superior y ser más competitivo. 
Tabla 1. Ejemplos de prioridades competitivas y sus definiciones

Fuente: Elaboración propia a partir de Miller y Roth, 2008; Díaz-Garrido, et al. 2011; Sarache, et al. 2011

\begin{tabular}{|c|l|}
\hline $\begin{array}{c}\text { Prioridades } \\
\text { Competitivas }\end{array}$ & \multicolumn{1}{|c|}{ Descripción } \\
\hline Costo & $\begin{array}{l}\text { Producción y distribución de productos a bajo costo. Esta relacionado } \\
\text { con una alta utilización de los recursos, alta productividad y bajos } \\
\text { niveles de costo de producción. }\end{array}$ \\
\hline Flexibilidad & $\begin{array}{l}\text { Habilidad de manejar cambios en el volumen y/o mezcla de producto. } \\
\text { Esta relacionado con habilidad de personalizar productos y entregas de } \\
\text { acuerdo a las especificaciones del cliente. }\end{array}$ \\
\hline Calidad & $\begin{array}{l}\text { Manufactura de productos con estándares de alta calidad y } \\
\text { desempeño. Esta relacionado con un alto desempeño del producto y } \\
\text { una alta conformidad del producto a las especificaciones de diseño, } \\
\text { seguridad y confiabilidad del producto. }\end{array}$ \\
\hline Entrega & $\begin{array}{l}\text { Entrega de productos confiable (a tiempo) y rápida (tiempo de entrega } \\
\text { corto). Esta relacionado con tiempos de producción cortos y habilidad } \\
\text { de entregar el producto final a tiempo. }\end{array}$ \\
\hline Innovación & $\begin{array}{l}\text { Diferenciación de los competidores en función de la innovación de sus } \\
\text { productos y procesos. Esta relacionado con caracteristicas y } \\
\text { funcionalidad innovadora en el producto y la tecnología usada, ofrecer } \\
\text { nuevos productos y uso de tecnología de punta. }\end{array}$ \\
\hline Medio Ambiente & $\begin{array}{l}\text { Cubrir las necesidades del cliente. Esta relacionado con servicio post- } \\
\text { venta, personalización de productos, soporte técnico del producto e } \\
\text { información para clientes y promesa de confianza. }\end{array}$ \\
\hline $\begin{array}{l}\text { Minimizar las repercusiones de la actividad productiva en el medio } \\
\text { ambiente. Esta relacionado con politicas de protección al ambiente a } \\
\text { traves de los procesos productivos y del producto en si. }\end{array}$ \\
\hline $\begin{array}{l}\text { Administración del conocimiento, creatividad, aprendizaje continuo, } \\
\text { desarrollo de habilidades para resolución de problemas, educación y } \\
\text { entrenamiento, investigación y desarrollo todo orientado a responder a } \\
\text { ciclos de vida de producto cada vez mas cortos. }\end{array}$ \\
\hline Clientow
\end{tabular}

Las capacidades de manufactura de una empresa en sí mismas no lograrán mejorar el desempeño de un sistema, sino las decisiones que se toman y las acciones que se realizan para cambiar las propiedades de las aéreas de decisión. Como se observa en la Tabla 2, estas áreas o categorías de decisión se agrupan en dos grandes áreas: decisiones estructurales y decisiones infraestructurales (Hayes y Wheelwright, 1984; Fine y Hax, 1985; Rudberg y Olhager, 2003; Hallgren y Olhager, 2006). De acuerdo a Vivares-Vergara, et al. 2014, las decisiones estructurales se caracterizan por su impacto de largo plazo por causa de la alta inversión que usualmente requieren y que afectan de manera significativa las capacidades del sistema de manufactura. En cambio las decisiones infraestructurales se enfocan en lograr que los procesos de administración de diversas áreas de la empresa presten un mejor apoyo a la función de manufactura. Son estas decisiones las que determinan qué recursos, procesos y rutinas se deben usar para alcanzar los objetivos de manufactura. Son estas elecciones las que determinan las características operativas del sistema de manufactura y que a su vez constituyen las capacidades de manufactura del sistema (Tan, et al. 2007). 
Tabla 2. Ejemplos de áreas de decisión y políticas disponibles Fuente: Elaboración propia a partir de Hallgren y Olhager, 2006

\begin{tabular}{|c|l|}
\hline Áreas de Decisión & \multicolumn{1}{|c|}{ Politicas Disponibles } \\
\hline Decisiones Estructurales & \\
Tecnología de proceso & Elección de proceso, Tecnología, Integración \\
Facilidades físicas & Tamaño, Localización, Enfoque \\
Capacidad & Cantidad, Tiempo, Incrementos \\
Integración vertical & Dirección, Extención, Balance \\
\hline Decisiones Infraestructurales & \\
Plan de manufactura y control & Diseño del sistema, Apoyo a las decisiones \\
Medición del desempeño & Métricas, Métodos de medición \\
Organización & Recursos humanos, Diseño \\
Calidad & Definición, Rol, Herramientas \\
\hline
\end{tabular}

De allí que el identificar cual es el impacto que tendrán estas decisiones y sus correspondientes acciones en el desempeño general del sistema es un tema de investigación recurrente en la literatura.

\subsection{Modelos teóricos de prioridades competitivas}

Krajewski y Ritzman en Awward, et al. (2013), definen las prioridades competitivas como "las dimensiones que el sistema de producción de una empresa debe poseer para apoyar las demandas de los mercados en los que la empresa desea competir". Como se explicó anteriormente, diferentes autores han identificado distintas prioridades competitivas. Como explican Díaz-Garrido y Martín-Peña (2007), el modelo de "tradeoff' (incompatibilidades por su traducción al español) introducido por Skinner (1969) consiste en ofrecer un tratamiento preferencial a una de las prioridades competitivas sobre el resto, debido a que, es imposible sobresalir en todas de manera simultánea y por lo tanto es necesario centrarse en una sacrificando equilibradamente el resto (Fine y Hax, 1985). Cada una de estas prioridades está sustentada por un grupo de componentes o dimensiones que deben ser medibles y que varían dependiendo de la industria o mercado.

Para identificar el trade-off existente entre las diferentes prioridades para un país, región o sector industrial especifico, varios autores han sugerido modelos de referencia que usan un análisis empírico de la percepción de directores, vicepresidentes o gerentes de empresa (Kathuria, 2000; Díaz-Garrido, et al. 2011; Rostek, 2012; Awward, et al. 2013) o de la opinión de expertos (Sarache, et al. 2012) sobre al nivel de importancia que tiene cada prioridad. Las respuestas obtenidas son luego ponderadas usando diferentes ecuaciones o algoritmos que permiten encontrar la importancia relativa o peso de cada componente o dimensión y a su vez de cada prioridad.

Una vez que estos pasos se han realizado, el orden jerárquico o modelo encontrado permite definir cuáles son las tareas que la unidad de manufactura debe de ejecutar bien para alcanzar los requisitos de mercado que le permitirán a la empresa ser competitiva (Porter, 1980; Martínez y Bititci, 2006), o en donde debe de enfocar más recursos (Kathuria, 2000; Boyer y Lewis, 2002). Tener congruencia entre las prioridades competitivas que se persiguen y las decisiones de manufactura que se toman para lograrlo (que combinado se vuelve las capacidades de manufactura de la empresa), permitirá a la compañía tener un rendimiento empresarial superior y ser más competitivo. 
Algunos ejemplos específicos del uso de estos modelos son los trabajos realizados para buscar desarrollar indicadores estratégicos que permitan medir la posición de las empresas con respecto a sus prioridades competitivas. Un ejemplo de esto es el trabajo de Díaz-Garrido, et al. (2011) que propone un indicador que permite posicionar a las empresas con respecto a sus prioridades competitivas (también llamadas capacidades de operación) y analiza la relación del mismo con los resultados empresariales. Otro ejemplo es el de Sarache, et al. (2011) que construye un procedimiento multicriterio para definir prioridades desde la óptica del cliente y mediante un indicador determinar el nivel de acercamiento a los deseos de los clientes en la industria de la confección. Adicionales ejemplos son los de Kroes y Ghosh (2010), Rostek (2012), Avella et al. (2001), Avella 2010, Cai y Yang (2014), Dangayach y Deshmukn (2006) y Swink y Nair (2007).

En el caso de Díaz-Garrido, et al. (2011) se usó una muestra de 1820 empresas con un $19.53 \%$ de respuesta (353 empresas) de varios sectores industriales en España y un instrumento (cuestionario) para identificar la importancia que le daban a diferentes prioridades competitivas en forma de variables multidimensionales y la fortaleza que tenían en ellas. El instrumento se construyó usando una escala Likert de 7 puntos para estudiar ciertos componentes. Los resultados del cuestionario fueron sometidos a varias pruebas para revisar la validez de cada constructo (correlación entre las dimensiones de una prioridad), consistencia interna del instrumento (Alfa de Cronbach), y los pesos asignados a cada componente (análisis exploratorio de factores).

En el caso de Sarache, et al. (2011) su metodología cambia ya que en lugar de utilizar encuestas para definir las prioridades competitivas (factores) y sus correspondientes dimensiones (componentes), se usó el método de expertos con 11 representantes de ventas de las empresas transnacionales (clientes). Los resultados obtenidos de las rondas preliminares pueden verse en la Tabla 3. La ponderación de factores y componentes en este caso se hizo usando un procedimiento que combinaba la comparación binaria entre factores y componentes usando la escala de Saaty y la ponderación a partir de las matrices obtenidas mediante un conjunto de ecuaciones matemáticas simultáneas. Luego se procedió a realizar una prueba de inconsistencia (calidad de la valoración del experto) y de concordancia (nivel de acuerdo entre juicios emitidos).

Sin embargo, Avella, et al. (2001) notó que hay ciertas empresas que tienden a alcanzar de forma simultánea niveles aceptables en todas las prioridades competitivas, debido a la implementación de ciertas prácticas tales como mejora continua, manufactura esbelta (Lean Manufacturing), administración de la calidad total (TQM), etc. Según ellos, en estas empresas no se aplica el modelo del trade-off sino el de capacidades acumuladas (cumulative capabilities) propuesto inicialmente por Ferdows y De Meyer (1990) y conocido como modelo cono de arena (sand cone) el cual fue luego generalizado por Noble (1995) y Flynn y Flynn (2004).

El modelo de cono de área sugiere que las empresas pueden construir una capacidad de manufactura sobre otra de manera secuencial de forma que pueden competir en la base de múltiples capacidades de operación de forma simultánea. El orden en que estas capacidades deben irse construyendo es parte de un debate (Nakane, 1986; Ferdows y De Meyer, 1990; Schmenner y Swink, 1998; Flynn y Flynn, 2004; Avella, et al. 2010), pero casi todos coinciden en que calidad debería de ser la primera capacidad a desarrollar. La 
idea es que la calidad sirve como punto de partida para ir desarrollando gradualmente las otras prioridades competitivas (Sarache, et al. 2011). Empresas que logran este tipo de nivel de competencia son conocidas como empresas de clase mundial.

\subsection{Identificación de factores y componentes}

Debido a que las prioridades competitivas son de naturaleza multidimensional, es necesario identificar cuáles son los componentes o dimensiones más importantes dentro de dichas prioridades. Para hacerlo se hace uso del procedimiento estadístico denominado análisis de componentes principales (PCA por sus siglas en ingles). De acuerdo a Abdi y Williams, (2010) PCA es una técnica multivariante que se usa para analizar variables cuantitativas dependientes posiblemente inter-correlacionadas entre sí. Su propósito es mostrar el patrón de similitud existente entre las variables, agrupándolas en variables ortogonales llamadas componentes principales. Mediante esta técnica es posible revelar la estructura interna de los datos, reducir el número de variables y crear una expresión cuyos componentes principales son la combinación lineal de las variables originales.

Terrádez (2014) explica que la idea detrás de esta técnica es que en un set de datos en donde hay variables que presentan altas correlaciones entre si, son pocos los factores que explicarán gran parte de la variabilidad existente. Aquellos factores que recojan la mayor proporción de variabilidad original son denominados componentes principales. Estos elementos se analizan usando una matriz factorial en donde los elementos son rotados usando el método de normalización Variamax en donde las columnas son los componentes principales y las filas las variables. En esta matriz los factores se identifican al obtener coeficientes factoriales cercanos a 1, tener coeficientes elevados en un solo factor y no tener factores con coeficientes similares. Paquetes de software tales como SPSS o Minitab tienen herramientas para reducir dimensiones mediante análisis de componentes principales.

Tabla 3. Ejemplo de una matriz de componentes rotados

Fuente: Tomado de Díaz-Garrido, et al. 2011

\begin{tabular}{|c|c|c|c|c|c|}
\hline & \multicolumn{5}{|l|}{ Factor } \\
\hline & 1 & 2 & 3 & 4 & 5 \\
\hline Importance of offering products quickly & 0.762 & 0.058 & 0.026 & 0.078 & 0.100 \\
\hline Importance of quick capacity increases given raised demand & 0.656 & 0.051 & 0.211 & -0.005 & 0.262 \\
\hline Importance of offering product when required by consumer & 0.634 & 0.236 & 0.135 & 0.146 & -0.050 \\
\hline Importance of facilitating making of orders and returns & 0.581 & 0.380 & 0.142 & 0.189 & -0.097 \\
\hline Importance of capacity to operate at different output levels & 0.546 & -0.130 & 0.364 & 0.242 & 0.301 \\
\hline Importance of offering complete and effective after-sales service & 0.057 & 0.695 & 0.059 & 0.115 & 0.022 \\
\hline Importance of providing customer with complete product information & 0.245 & 0.675 & 0.020 & 0.045 & 0.222 \\
\hline Importance of offering personalised service & 0.147 & 0.638 & 0.270 & 0.119 & -0.127 \\
\hline Importance of manufacturing wide range of products & 0.283 & 0.074 & 0.752 & 0.014 & 0.061 \\
\hline Importance of ability of machinery to manufacture different products & 0.183 & -0.070 & 0.742 & 0.182 & 0.040 \\
\hline Importance of manufacturing products with multiple characteristics & -0.051 & 0.434 & 0.678 & 0.059 & -0.095 \\
\hline Importance of quick changes in product design & -0.129 & 0.383 & 0.583 & -0.130 & 0.242 \\
\hline Importance of manufacturing products that respect environment & 0.116 & 0.119 & 0.061 & 0.847 & 0.135 \\
\hline Importance of non-contaminating production process & 0.196 & 0.127 & 0.084 & 0.819 & 0.169 \\
\hline Importance of reducing product cost & -0.020 & -0.212 & 0.089 & 0.056 & 0.594 \\
\hline Importance of providing lasting and reliable products & -0.026 & 0.504 & 0.053 & -0.037 & 0.590 \\
\hline Importance of providing defect-free products & 0.149 & 0.022 & 0.003 & 0.274 & 0.548 \\
\hline Importance of providing products complying with design specifications & 0.143 & 0.189 & 0.009 & 0.064 & 0.502 \\
\hline
\end{tabular}

\section{Metodología}

En vista que el propósito de este estudio es desarrollar un instrumento que identifique las prioridades competitivas del sector maquilador, el tipo de investigación será exploratorio, no experimental con un diseño transeccional exploratorio. Debido a que las prioridades 
competitivas (variables) se conciben como elementos multidimensionales, estas dimensiones deben determinarse. Para hacerlo; inicialmente se hará un análisis comparativo de los principales artículos que hay en la literatura tratando la temática de prioridades competitivas, buscando identificar las prioridades competitivas mencionadas y sus respectivos componentes propuestos.

Usando este análisis de identificación se seleccionarán las prioridades (factores) y los elementos (componentes) que a nuestro criterio deberían usarse para determinar las prioridades competitivas del sector maquilador. Usando estos insumos, se buscará la opinión de un grupo de expertos para determinar qué elementos incluir en el instrumento a producir. Usando las sugerencias y comentarios de los expertos se planteará el instrumento final a ser usado.

Finalmente se propondrá una metodología para usar el instrumento (colocar y analizar) en el proceso de identificar las prioridades competitivas del sector maquilador de San Pedro Sula.

\section{Resultados y análisis}

\subsection{Instrumento propuesto}

A partir de los artículos escritos por Kroes y Ghosh (2010); Avella et al. (2001), Avella 2010, Díaz-Garrido et al. (2010), Sarache et al. (2012), Cai y Yang (2014), Dangayach y Deshmukn (2006), Swink y Nair (2007), Hallgren (2007) y Khanchanapong et al. (2014) se hizo una tabla en donde se compararon y contrastaron los componentes de cada una de las prioridades competitivas identificada (Tabla 4). Producto de este análisis bibliográfico se encontraron que son 7 las prioridades competitivas más utilizadas, de las cuales 4 son las mencionadas en todos los artículos y el resto apareciendo solo en algunos de ellos (Figura 2).

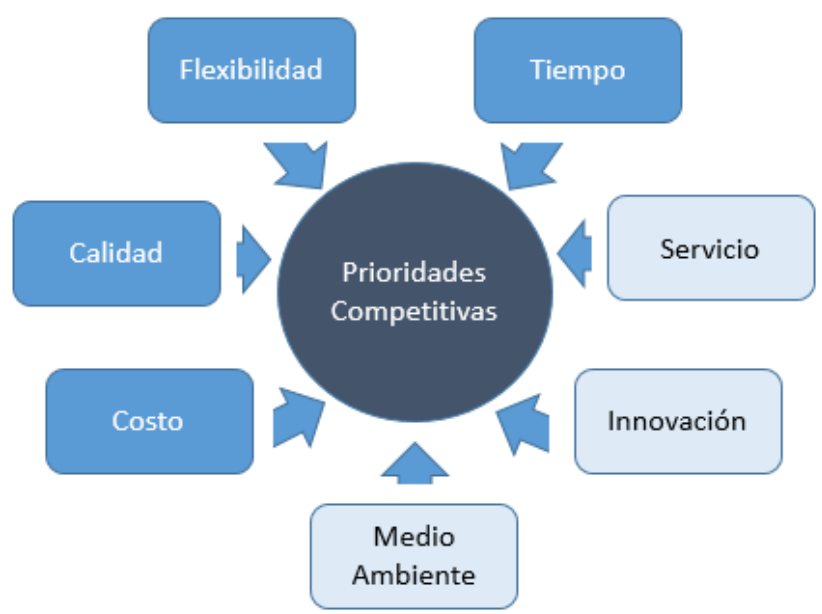

Figura 2. Modelo investigativo de las prioridades competitivas 


\section{(it)|unitec}

Tabla 4. Prioridades competitivas y sus componentes de acuerdo a diferentes autores

\begin{tabular}{|c|c|c|c|c|c|c|c|c|c|c|}
\hline $\begin{array}{l}\text { Prioridades } \\
\text { Competitivas }\end{array}$ & $\begin{array}{c}\text { Kroes y Ghosh, } \\
2010\end{array}$ & $\begin{array}{c}\text { Avella, et al. } \\
2001\end{array}$ & Avella, 2010 & $\begin{array}{c}\text { Díaz-Garrido, } \\
\text { et al. } 2011\end{array}$ & $\begin{array}{c}\text { Sarache, et al. } \\
2012\end{array}$ & Cai y Yang, 2014 & $\begin{array}{c}\text { Dangayach y } \\
\text { Deshmukn, } 2006\end{array}$ & $\begin{array}{c}\text { Swink y Nair, } \\
2007\end{array}$ & Hallgren, 2007 & $\begin{array}{c}\text { Khanchanap } \\
\text { ong, et al. } \\
2014 \\
\end{array}$ \\
\hline \multirow{6}{*}{ Costo } & $\begin{array}{l}\text { Alta capacidad de } \\
\text { utilización }\end{array}$ & Bajo costo & $\begin{array}{l}\text { Reducción de costo } \\
\text { de manufactura }\end{array}$ & Bajo costo & Costo & $\begin{array}{l}\text { Reducción de costo } \\
\text { de producción }\end{array}$ & Bajo costo & $\begin{array}{l}\text { Costo inicial de } \\
\text { compra }\end{array}$ & $\begin{array}{l}\text { Costo unitario de } \\
\text { manufactura }\end{array}$ & $\begin{array}{c}\text { Costo de } \\
\text { producción bajo }\end{array}$ \\
\hline & $\begin{array}{l}\text { Alta productividad } \\
\text { laboral }\end{array}$ & & $\begin{array}{l}\text { Incremento de } \\
\text { productividad } \\
\text { laboral }\end{array}$ & & & $\begin{array}{l}\text { Reducción de costo al } \\
\text { aumentar } \\
\text { productividad }\end{array}$ & & $\begin{array}{l}\text { Costo general de } \\
\text { manufactura }\end{array}$ & $\begin{array}{l}\text { Rotación de } \\
\text { inventarios }\end{array}$ & $\begin{array}{l}\text { Precios } \\
\text { competitivos }\end{array}$ \\
\hline & $\begin{array}{l}\text { Bajo costo de } \\
\text { inventario }\end{array}$ & & $\begin{array}{l}\text { Incremento en } \\
\text { capacidad o } \\
\text { utilización de } \\
\text { equipo }\end{array}$ & & & $\begin{array}{l}\text { Reducción de costo al } \\
\text { mejorar la utilización } \\
\text { de la capacidad }\end{array}$ & & & $\begin{array}{l}\text { Utilización de la } \\
\text { capacidad }\end{array}$ & $\begin{array}{l}\text { Eficiencia de la } \\
\text { producción }\end{array}$ \\
\hline & $\begin{array}{l}\text { Baja costo de } \\
\text { producción / } \\
\text { manufactura }\end{array}$ & & $\begin{array}{l}\text { Reducción de nivel } \\
\text { de inventario }\end{array}$ & & & $\begin{array}{l}\text { Reducción de costos } \\
\text { mediante reducción } \\
\text { de tiempo de ciclos } \\
\text { de producción }\end{array}$ & & & Rendimiento & $\begin{array}{l}\text { Rotación de } \\
\text { inventarios }\end{array}$ \\
\hline & & & & & & $\begin{array}{c}\text { Reducción de costo } \\
\text { de inventarios }\end{array}$ & & & $\begin{array}{c}\text { Precio de venta del } \\
\text { producto }\end{array}$ & \\
\hline & & & & & & & & & Precio de mercado & \\
\hline \multirow{4}{*}{ Calidad } & $\begin{array}{l}\text { Alta conformidad de } \\
\text { producto final a } \\
\text { especificaciones de } \\
\text { diseño }\end{array}$ & $\begin{array}{l}\text { Productos libre } \\
\text { de defecto (o } \\
\text { bajas tasas de } \\
\text { defecto) }\end{array}$ & $\begin{array}{c}\text { Mejorar la } \\
\text { conformidad de las } \\
\text { especificaciones } \\
\text { con las } \\
\text { especificaciones de } \\
\text { diseño }\end{array}$ & $\begin{array}{l}\text { Desempeño del } \\
\text { producto }\end{array}$ & Calidad comparada & $\begin{array}{l}\text { Calidad de } \\
\text { desempeño }\end{array}$ & $\begin{array}{l}\text { Conformidad de la } \\
\text { calidad }\end{array}$ & $\begin{array}{l}\text { Características del } \\
\text { producto }\end{array}$ & Costo de re-trabajo & $\begin{array}{l}\text { Durabilidad del } \\
\text { producto }\end{array}$ \\
\hline & $\begin{array}{l}\text { Alto rendimiento del } \\
\text { producto }\end{array}$ & $\begin{array}{l}\text { Calidad percibida } \\
\text { por el cliente }\end{array}$ & $\begin{array}{l}\text { Oferta de calidad } \\
\text { consistente y fiable }\end{array}$ & Conformidad & $\begin{array}{l}\text { Posibilidad de } \\
\text { rechazo }\end{array}$ & $\begin{array}{l}\text { Durabilidad del } \\
\text { producto }\end{array}$ & $\begin{array}{l}\text { Fiabilidad del } \\
\text { producto }\end{array}$ & $\begin{array}{l}\text { Durabilidad del } \\
\text { producto }\end{array}$ & $\begin{array}{c}\text { Porcentaje de } \\
\text { inspección de } \\
\text { calidad superada }\end{array}$ & $\begin{array}{l}\text { Fiabilidad del } \\
\text { producto }\end{array}$ \\
\hline & $\begin{array}{l}\text { Alta fiabilidad de } \\
\text { producto }\end{array}$ & $\begin{array}{l}\text { Productos } \\
\text { durables }\end{array}$ & $\begin{array}{l}\text { Proporcionar } \\
\text { productos de alto } \\
\text { rendimiento }\end{array}$ & $\begin{array}{l}\text { Fiabilidad del } \\
\text { producto }\end{array}$ & $\begin{array}{c}\text { Fiabilidad de la } \\
\text { entrega }\end{array}$ & $\begin{array}{l}\text { Conformidad de la } \\
\text { calidad }\end{array}$ & $\begin{array}{l}\text { Desempeño del } \\
\text { producto }\end{array}$ & $\begin{array}{l}\text { Conformidad del } \\
\text { producto }\end{array}$ & $\begin{array}{c}\text { Costo del control de } \\
\text { la calidad }\end{array}$ & $\begin{array}{l}\text { Desempeño del } \\
\text { producto }\end{array}$ \\
\hline & $\begin{array}{l}\text { Alta seguridad del } \\
\text { producto }\end{array}$ & & $\begin{array}{l}\text { Ofrecer productos } \\
\text { duraderos y fiables }\end{array}$ & & & $\begin{array}{l}\text { Reducción de } \\
\text { defectos }\end{array}$ & $\begin{array}{l}\text { Durabilidad del } \\
\text { producto }\end{array}$ & $\begin{array}{l}\text { Fiabilidad del } \\
\text { producto }\end{array}$ & $\begin{array}{c}\text { Conformidad con las } \\
\text { especificaciones } \\
\text { acordadas }\end{array}$ & $\begin{array}{l}\text { Calidad global } \\
\text { del producto } \\
\text { según la } \\
\text { percepción del } \\
\text { cliente }\end{array}$ \\
\hline
\end{tabular}




\begin{tabular}{|c|c|c|c|c|c|c|c|c|c|c|}
\hline & $\begin{array}{l}\text { Kroes y Ghosh, } \\
2010\end{array}$ & $\begin{array}{l}\text { Avella, et al. } \\
2001\end{array}$ & Avella, 2010 & $\begin{array}{l}\text { Díaz-Garrido, } \\
\text { et al. } 2011\end{array}$ & $\begin{array}{l}\text { Sarache, et al. } \\
\qquad 2012\end{array}$ & Cai y Yang, 2014 & $\begin{array}{c}\text { Dangayach y } \\
\text { Deshmukn, } 2006\end{array}$ & $\begin{array}{c}\text { Swink y Nair, } \\
2007\end{array}$ & Hallgren, 2007 & $\begin{array}{c}\text { Khanchanap } \\
\text { ong, et al. } \\
2014\end{array}$ \\
\hline & $\begin{array}{l}\text { Pronta resolución de } \\
\text { quejas/dudas de los } \\
\text { clientes }\end{array}$ & & $\begin{array}{c}\text { Fabricación con } \\
\text { tasas constantes de } \\
\text { bajos defectos } \\
\text { (reducción de tasa } \\
\text { de defectos) } \\
\end{array}$ & & & & & $\begin{array}{l}\text { Rendimiento de la } \\
\text { calidad del } \\
\text { producto }\end{array}$ & $\begin{array}{l}\text { Desempeño del } \\
\text { producto }\end{array}$ & $\begin{array}{l}\text { Conformidad a } \\
\text { especificaciones }\end{array}$ \\
\hline & $\begin{array}{l}\text { Facilidad (costo y } \\
\text { tiempo) de reparar } \\
\text { un producto }\end{array}$ & & & & & & & & & \\
\hline & $\begin{array}{c}\text { Producto de alta } \\
\text { durabilidad (larga } \\
\text { vida) }\end{array}$ & & & & & & & & & \\
\hline \multirow{5}{*}{ Flexibilidad } & \begin{tabular}{|c|} 
Habilidad de ajustar \\
capacidad y/o \\
volumen \\
efectivamente dentro \\
de un periodo corto \\
de tiempo
\end{tabular} & $\begin{array}{l}\text { Cambios rápidos } \\
\text { en los diseños } \\
\text { actuales }\end{array}$ & $\begin{array}{l}\text { Hacer cambios de } \\
\text { diseño } \\
\text { rápidamente }\end{array}$ & $\begin{array}{l}\text { Expansión } \\
\text { (incrementos } \\
\text { rápidos en } \\
\text { capacidad) }\end{array}$ & $\begin{array}{l}\text { Flexibilidad para } \\
\text { cambios inesperados }\end{array}$ & $\begin{array}{l}\text { Habilidad de } \\
\text { introducir nuevos } \\
\text { productos }\end{array}$ & $\begin{array}{c}\text { Cambios en la mezcla } \\
\text { de productos }\end{array}$ & $\begin{array}{l}\text { Número de nuevos } \\
\text { productos } \\
\text { introducidos cada } \\
\text { año }\end{array}$ & $\begin{array}{c}\text { Tiempo/costo de } \\
\text { preparación (set up) }\end{array}$ & \begin{tabular}{|l} 
Cambio fácil del \\
volumen de \\
producción de \\
un proceso de \\
manufactura
\end{tabular} \\
\hline & \begin{tabular}{|c} 
Capacidad de ajustar \\
las entregas para \\
satisfacer las \\
necesidades del \\
cliente \\
\end{tabular} & $\begin{array}{c}\text { Rápida } \\
\text { introducción de } \\
\text { nuevos } \\
\text { productos }\end{array}$ & $\begin{array}{l}\text { Introducir nuevos } \\
\text { productos } \\
\text { rápidamente }\end{array}$ & \begin{tabular}{|c|} 
Variaciones en \\
volumen de \\
producción (ajustes \\
rápidos en \\
capacidad) \\
\end{tabular} & $\begin{array}{l}\text { Flexibilidad del } \\
\text { portafolio de familia } \\
\text { de productos }\end{array}$ & $\begin{array}{l}\text { Flexibilidad de } \\
\text { volumen }\end{array}$ & $\begin{array}{l}\text { Personalización del } \\
\text { producto }\end{array}$ & $\begin{array}{l}\text { Tiempo para } \\
\text { introducir nuevos } \\
\text { productos }\end{array}$ & $\begin{array}{l}\text { Duración del } \\
\text { programa fijo de } \\
\text { producción }\end{array}$ & \begin{tabular}{|c|} 
Construir \\
diferentes \\
productos en la \\
misma planta al \\
mismo tiempo \\
\end{tabular} \\
\hline & $\begin{array}{l}\text { Habilidad de } \\
\text { personalizar los } \\
\text { productos para } \\
\text { satisfacer las } \\
\text { necesidades del } \\
\text { cliente }\end{array}$ & $\begin{array}{l}\text { Cambios rápidos } \\
\text { en volumen }\end{array}$ & $\begin{array}{l}\text { Hacer cambios } \\
\text { rápidos en } \\
\text { volumen }\end{array}$ & $\begin{array}{c}\text { Cambios en diseño } \\
\text { de producto }\end{array}$ & $\begin{array}{l}\text { Flexibilidad en } \\
\text { tamaño de la orden }\end{array}$ & $\begin{array}{l}\text { Habilidad de hacer } \\
\text { cambios rápidos de } \\
\text { diseño }\end{array}$ & Cambios de volumen & $\begin{array}{l}\text { Habilidad de } \\
\text { responder a } \\
\text { cambios en } \\
\text { requerimientos de } \\
\text { entrega }\end{array}$ & $\begin{array}{l}\text { Cantidad de } \\
\text { capacidad de } \\
\text { operación }\end{array}$ & $\begin{array}{c}\text { Cambio rápido } \\
\text { de un producto } \\
\text { al otro }\end{array}$ \\
\hline & \begin{tabular}{|c|} 
Capacidad para \\
realizar cambios de \\
diseño en el producto \\
una vez iniciada \\
producción
\end{tabular} & $\begin{array}{l}\text { Cambios rápidos } \\
\text { en mezcla de } \\
\text { producto }\end{array}$ & $\begin{array}{l}\text { Realizar rápidos } \\
\text { cambios de mezcla } \\
\text { de producto }\end{array}$ & $\begin{array}{l}\text { Amplio rango de } \\
\text { productos }\end{array}$ & $\begin{array}{l}\text { Flexibilidad de } \\
\text { portafolio de telas }\end{array}$ & $\begin{array}{l}\text { Amplia línea de } \\
\text { productos }\end{array}$ & Cambios de diseño & $\begin{array}{l}\text { Posibilidad de } \\
\text { ajustar los } \\
\text { volúmenes de } \\
\text { producción }\end{array}$ & Rango de productos & $\begin{array}{c}\text { Cambio fácil de } \\
\text { los productos a } \\
\text { una necesidad } \\
\text { específica de un } \\
\text { cliente }\end{array}$ \\
\hline & $\begin{array}{c}\text { Ofrecer un gran } \\
\text { número de } \\
\text { características de } \\
\text { producto u opciones }\end{array}$ & $\begin{array}{l}\text { Amplia línea de } \\
\text { productos }\end{array}$ & $\begin{array}{l}\text { Ofrecer un alto } \\
\text { grado de variedad } \\
\text { de producto } \\
\text { (amplia línea de } \\
\text { productos) }\end{array}$ & $\begin{array}{c}\text { Amplia variedad de } \\
\text { productos } \\
\text { (productos no- } \\
\text { estandarizados) }\end{array}$ & & $\begin{array}{l}\text { Posibilidad de } \\
\text { cambiar rápidamente } \\
\text { la mezcla de } \\
\text { productos }\end{array}$ & & $\begin{array}{l}\text { Posibilidad de } \\
\text { personalizar } \\
\text { productos }\end{array}$ & $\begin{array}{c}\text { Número de } \\
\text { productos ofrecidos }\end{array}$ & \\
\hline
\end{tabular}




\begin{tabular}{|c|c|c|c|c|c|c|c|c|c|c|}
\hline & $\begin{array}{c}\text { Kroes y Ghosh, } \\
2010\end{array}$ & $\begin{array}{l}\text { Avella, et al. } \\
2001\end{array}$ & Avella, 2010 & $\begin{array}{l}\text { Díaz-Garrido, } \\
\text { et al. } 2011\end{array}$ & $\begin{array}{l}\text { Sarache, et al. } \\
2012\end{array}$ & Cai y Yang, 2014 & $\begin{array}{c}\text { Dangayach y } \\
\text { Deshmukn, } 2006\end{array}$ & $\begin{array}{l}\text { Swink y Nair, } \\
2007\end{array}$ & Hallgren, 2007 & $\begin{array}{c}\text { Khanchanap } \\
\text { ong, et al. } \\
2014\end{array}$ \\
\hline & $\begin{array}{l}\text { Producir gran } \\
\text { variedad de } \\
\text { productos }\end{array}$ & & $\begin{array}{l}\text { Ajustar mezcla de } \\
\text { producto }\end{array}$ & $\begin{array}{l}\text { Mezcla de } \\
\text { producto }\end{array}$ & & & & $\begin{array}{c}\text { Habilidad de } \\
\text { producir un rango } \\
\text { de productos }\end{array}$ & $\begin{array}{c}\text { Capacidad de } \\
\text { manejar cambios en } \\
\text { volumen y mezcla }\end{array}$ & \\
\hline & $\begin{array}{l}\text { Alta flexibilidad de } \\
\text { producción para } \\
\text { permitir la } \\
\text { introducción de } \\
\text { nuevos productos }\end{array}$ & & & & & & & & & \\
\hline \multirow{6}{*}{ Tiempo } & $\begin{array}{l}\text { Tiempos cortos de } \\
\text { cambio/preparación }\end{array}$ & Entregas rápidas & $\begin{array}{l}\text { Proveer entregas } \\
\text { rápidas }\end{array}$ & Entregas rápidas & Tiempo de entrega & Velocidad de entrega & Entrega confiable & \begin{tabular}{|c|} 
Velocidad de \\
entrega (tiempo \\
transcurrido corto)
\end{tabular} & Tiempo de entrega & $\begin{array}{l}\text { Tiempo de } \\
\text { procuramiento }\end{array}$ \\
\hline & $\begin{array}{l}\text { Tiempo de ciclo de } \\
\text { desarrollo de } \\
\text { producto corto }\end{array}$ & $\begin{array}{l}\text { Entregas a } \\
\text { tiempo o } \\
\text { confiables }\end{array}$ & $\begin{array}{c}\text { Cumplir con las } \\
\text { promesas o } \\
\text { compromisos de } \\
\text { entrega } \\
\end{array}$ & Entregas a tiempo & $\begin{array}{l}\text { Tiempo de respuesta } \\
\text { a un cliente que pide } \\
\text { información }\end{array}$ & Fiabilidad de entrega & Velocidad de entrega & $\begin{array}{c}\text { Disponibilidad de } \\
\text { entrega (artículos } \\
\text { estarán en stock al } \\
\text { momento del } \\
\text { pedido) } \\
\end{array}$ & Entregas a tiempo & $\begin{array}{l}\text { Tiempo de } \\
\text { fabrircación }\end{array}$ \\
\hline & $\begin{array}{l}\text { Tiempos de ciclo de } \\
\text { producción cortos }\end{array}$ & & $\begin{array}{l}\text { Reducir tiempo de } \\
\text { manufactura }\end{array}$ & $\begin{array}{c}\text { Pedidos y } \\
\text { devoluciones }\end{array}$ & $\begin{array}{c}\text { Tiempo de respuesta } \\
\text { a una solicitud de } \\
\text { cotización }\end{array}$ & & & \begin{tabular}{|c|} 
Fiabilidad de la \\
entrega (entregado \\
en fecha acordada)
\end{tabular} & $\begin{array}{l}\text { Disponibilidad de } \\
\text { stock }\end{array}$ & $\begin{array}{l}\text { Velocidad de } \\
\text { entrega }\end{array}$ \\
\hline & $\begin{array}{l}\text { Tiempo de } \\
\text { producción cortos }\end{array}$ & & & & & & & $\begin{array}{l}\text { Exactitud de la } \\
\text { entrega (se } \\
\text { entregaron los } \\
\text { items correctos) }\end{array}$ & $\begin{array}{l}\text { Tiempo de } \\
\text { producción }\end{array}$ & \\
\hline & $\begin{array}{l}\text { Introducción rápida } \\
\text { de nuevos productos }\end{array}$ & & & & & & & & $\begin{array}{l}\text { Precisión del estado } \\
\text { del inventario }\end{array}$ & \\
\hline & $\begin{array}{c}\text { Capacidad de } \\
\text { entregar a tiempo }\end{array}$ & & & & & & & & $\begin{array}{c}\text { Fiabilidad de los } \\
\text { plazos de entrega } \\
\text { interna }\end{array}$ & \\
\hline \multirow{3}{*}{ Servicio } & & & & Servicio post-venta & Asistencia técnica & & & & & \\
\hline & & & & $\begin{array}{c}\text { Necesidades del } \\
\text { cliente }\end{array}$ & & & & & & \\
\hline & & & & Información & & & & & & \\
\hline Ambiente & & & $\begin{array}{c}\text { Hacer productos } \\
\text { amigables al } \\
\text { ambiente }\end{array}$ & $\begin{array}{l}\text { Protection of } \\
\text { environment } \\
\text { (production } \\
\text { process) }\end{array}$ & & & & & & \\
\hline
\end{tabular}




\begin{tabular}{|c|c|c|c|c|c|c|c|c|c|c|}
\hline & $\begin{array}{c}\text { Kroes y Ghosh, } \\
2010\end{array}$ & $\begin{array}{l}\text { Avella, et al. } \\
2001\end{array}$ & Avella, 2010 & $\begin{array}{c}\text { Díaz-Garrido, } \\
\text { et al. } 2011\end{array}$ & $\begin{array}{l}\text { Sarache, et al. } \\
\quad 2012\end{array}$ & Cai y Yang, 2014 & $\begin{array}{c}\text { Dangayach y } \\
\text { Deshmukn, } 2006\end{array}$ & $\begin{array}{l}\text { Swink y Nair, } \\
2007\end{array}$ & Hallgren, 2007 & $\begin{array}{c}\text { Khanchanap } \\
\text { ong, et al. } \\
2014\end{array}$ \\
\hline & & & $\begin{array}{l}\text { Utilización de } \\
\text { procesos de } \\
\text { producción } \\
\text { amigables al medio } \\
\text { ambiente }\end{array}$ & $\begin{array}{l}\text { Protección del } \\
\text { medio ambiente } \\
\text { (productos) }\end{array}$ & & & & & & \\
\hline & & & $\begin{array}{c}\text { Prevenir incidentes } \\
\text { ambientales }\end{array}$ & & & & & & & \\
\hline & & & $\begin{array}{c}\text { Proporcionar a la } \\
\text { empresa con una } \\
\text { imagen ambiental } \\
\text { positiva } \\
\end{array}$ & & & & & & & \\
\hline \multirow{6}{*}{ Innovación } & $\begin{array}{l}\text { Diferenciación de la } \\
\text { tecnología de } \\
\text { producto de los } \\
\text { competidores }\end{array}$ & & & & & & Nuevos productos & & & \\
\hline & $\begin{array}{c}\text { Características de } \\
\text { producto y } \\
\text { funcionalidad } \\
\text { innovadoras } \\
\end{array}$ & & & & & & & & & \\
\hline & $\begin{array}{c}\text { Ofrecer nuevas } \\
\text { innovaciones de } \\
\text { producto }\end{array}$ & & & & & & & & & \\
\hline & $\begin{array}{l}\text { Venta de productos } \\
\text { que están en las } \\
\text { etapas introductorias } \\
\text { o de crecimiento del } \\
\text { ciclo de vida de } \\
\text { producto }\end{array}$ & & & & & & & & & \\
\hline & $\begin{array}{l}\text { Uso de tecnologías } \\
\text { de producto / } \\
\text { tecnologías de } \\
\text { proceso }\end{array}$ & & & & & & & & & \\
\hline & $\begin{array}{l}\text { La tecnología de } \\
\text { producto actual } \\
\text { representa un gran } \\
\text { avance sobre la } \\
\text { tecnología de } \\
\text { producto anterior }\end{array}$ & & & & & & & & & \\
\hline
\end{tabular}


La información recopilada en la Tabla 4 fue presentada a un set de expertos con amplia experiencia en la industria maquiladora, así como en el sector académico para que determinaran que elementos deberían componer cada factor que se usaría en un instrumento a aplicarse a gerentes de planta. El método usado para procesar los resultados obtenidos fue una variación del método Delphy, debido a la falta de tiempo para recibir la respuesta de todos los expertos contactados y de estabilizar las respuestas recibidas.

Usando las respuestas recibidas, se tomó la opinión de los expertos y se colocaron en el instrumento aquellos elementos en los que hubo acuerdo total o parcial de criterios. Debido a los desafíos para buscar el consenso entre los expertos en aquellos elementos que había discrepancia, estos fueron agregados al instrumento pero aparecen en color rojo. El instrumento fue preparado basado en una escala Likert de 1 a 5 y puede verse en el Anexo A al final de este documento

\subsection{Metodología de aplicación y análisis del instrumento}

Una vez que la validez del contenido haya sido totalmente determinada al completar de implementar el método Delphi, la metodología que se sugiere para aplicar y analizar el instrumento es la siguiente:

1. Colocar instrumento a una muestra piloto de gerentes de planta de maquiladoras para obtener opiniones que permitan mejorar la redacción de los elementos del instrumento, especialmente en términos técnicos.

2. Usando la información obtenida de la muestra piloto realizar un análisis factorial (análisis de componentes principales) para validar los constructos, asegurándonos que los elementos usados en efecto pertenecen a cada factor.

3. Usando la información obtenida de la muestra piloto realizar un análisis de fiabilidad del instrumento mediante el cálculo del alfa de Cronbach (coeficiente de consistencia interna) para cada factor y para todo el instrumento.

4. Realizar los ajustes que sean necesarios en el instrumento (cambiar redacción y cambiar o quitar elementos) y aplicar la encuesta al resto de los gerentes de la muestra seleccionada.

5. Realizar el análisis factorial de todos los datos para obtener los pesos de cada elemento dentro de cada factor (ver valores en negrita en ejemplo en Tabla 3).

6. Al multiplicar el valor obtenido en cada elemento (valor de 1 a 5) por los pesos encontrados en el análisis factorial y luego realizar la sumatoria de dichos productos por cada factor, es posible obtener un valor acumulado por de cada factor. Los factores más altos obtenidos son las prioridades competitivas más altas de la industria.

\section{Conclusiones}

Uno de los desafíos más grandes que tiene la industria maquiladora es la de aumentar su competitividad para poder mantenerse en el mercado. Para poder hacerlo, los gerentes necesitan tomar decisiones operacionales que permitan mejorar su desempeño y competitividad. El identificar cuáles son las prioridades competitivas que demandan el 
mercado y cuáles son las prioridades que actualmente está persiguiendo la compañía, permitirá alinear la estrategia de manufactura de forma que se pueda responder apropiadamente a las demandas del mercado y mantenerse de esta forma competitivos.

En este estudio se ha revisado de forma extensa la literatura relacionada con las prioridades competitivas. Además de la revisión bibliográfica necesaria para enmarcar el tema, se ha realizado un análisis comparativo de 10 artículos para identificar los factores (prioridades) y sus componentes (elementos) mas usados. Esta información fue luego analizada por un panel de expertos y resumida a los elementos más importantes. Este insumo fue a su vez usado para construir una propuesta de instrumento a ser usada para identificar las prioridades competitivas de la industria maquiladora en San Pedro Sula. Finalmente se presentó una metodología para aplicar el instrumento, validarlo y analizarlo.

Se sugiere terminar el análisis de expertos para reducir la cantidad de componentes que actualmente se tienen en el instrumento (elementos en color rojo). También se sugiere convertir el instrumento en una encuesta electrónica que sea más fácil de aplicar y analizar. Finalmente se sugiere proponer una forma de usar indicadores claves de rendimiento (key performance indicators) para capturar la información que los componentes tratan de medir y de esta forma hacer la medición más confiable y que la misma no dependa de la percepción de alguien, sino de valores reales medibles.

\section{Bibliografía}

Abdi, H., \& Williams, L. J. (2010). Principal component analysis. Wiley Interdisciplinary Reviews: Computational Statistics, Vol. 2, N. 4, 433-459.

Alonso, J., Carrillo, J., \& Contreras, O. (2002). Aprendizaje tecnológico en las maquiladoras del norte de México. Revista Frontera Norte, Vol. 14, N. 27, 43-82.

Álvarez Gil, M. J., Burgos Jiménez, J. d., \& Céspedes Lorente, J. J. (2001). Un análisis exploratorio de las estrategias medioambientales y el contexto organizativo de los hoteles españoles. Cuadernos de Economía y Dirección de la Empresa, Vol. 8, 532.

Avella, L., Fernández, E., \& Vásquez, C. J. (2001). Analysis of manufacturing strategy as an explanatory factor of competitiveness in the large Spanish industrial firm. International Journal of Production Economics, Vol. 72, 139 - 157.

Avella, L., Vazquez-Bustelo, D., \& Fernandez, E. (2011). Cumulative manufacturing capabilities: An extended model and new empirical evidence. International Journal of Production Research, Vol. 49, N. 3, 707-729.

Awwad, A. S., Al Khattab, A. A., \& Anchor, J. R. (2013). Competitive Priorities and Competitive Advantage in Jordanian Manufacturing. Journal of Service Science and Management, Vol. 6, 69-79.

Boyer, K. K., \& Lewis, M. W. (2002). Competitive priorities: investigating the need for trade-offs in operations strategy. Production and Operations Management, Vol. 11, N. 1, 9-20. 
Buttler, T. W., \& Leong, G. K. (2000). The impact of operations competitive priorities on hospital performance. Health Care Management Science, Vol 3, N. 3, 227-235.

Comisión Económica para América Latina y el Caribe (CEPAL). (2004). La competitividad de la industria maquiladora de exportación en Honduras. Condiciones y retos ante el CAFTA. México, D.F.: Naciones Unidas.

Comisión Económica para América Latina y el Caribe (CEPAL). (2005). El medio ambiente y la maquila en México: un problema ineludible. México, D.F:: Naciones Unidas.

Corbett, C., \& Van Wassenhove, L. (1993). Trade-offs? What trade-offs? Competence and competitiveness in manufacturing strategy. California Management Review, Vol. 35, N. 4, 107-122.

Cozzarin, B. P. (2006). Are world-first innovations conditional on economic performance? Technovation, Vol. 26, N. 9, 1017-1028.

Da Silveira, G., \& Slack, N. (2001). Exploring the trade-off concept. International Journal of Operations \& Production Management, Vol. 21, N. 7, 949-964.

Dangayach, G. S., \& Deshmukh, S. G. (2006). An exploratory study of manufacturing strategy practices of machinery manufacturing companies in India. The International Journal of Management Science, Vol. 34, N. 3, 254-273.

Davis, M. M., Aquilano, N. J., \& Chase, R. B. (2001). Fundamentos de dirección de operaciones, 3ra edición. Madrid: McGraw-Hill.

De Carolis, D. M. (2003). Competencies and imitability in the pharmaceutical industry: An analysis of their relationship with firm performance. Journal of Management, Vol. 29, N. 1, 27-50.

Díaz-Garrido, E., Martín-Peña, M. L., \& Sánchez-López, J. M. (2011). Competitive priorities in operations: Development of an indicator of strategic position. CIRP Journal of Manufacturing Science and Technology, Vol. 4, 118-125.

Dornier, P.-P., Ernst, R., Fender, M., \& Kouvelis, P. (1998). Global operations and logistics: Text and cases. New York: John Wiley \& Sons Inc.

Ferdows, K., \& De Meyer, A. (1990). Lasting improvements in manufacturing performance: In search of a new theory. Journal of Operations Management, Vol. 9, N. 2, 168-184.

Fine, C. H., \& Hax, A. C. (1985). Manufacturing strategy: A methodology and an illustration. Interfaces, Vol. 15, N. 6, 28-46.

Flynn, B. B., \& Flynn, E. J. (2004). An exploratory study of the nature of cumulative capabilities. Journal of Operations Management, Vol. 22, N. 5, 439-457.

Greasley, A. (2009). Operations Management, 2da edición. Chichester: John Wiley.

Größler, A., \& Grübner, A. (2006). An empirical model of the relationship between manufacturing capabilities. International Journal of Operations \& Production Management, Vol 26, N. 5, 458-485. 
Hallgren, M. (2007). Manufacturing Strategy, capabilities and performance (tesis doctoral). Linköping: Linköping Studies in Science and Technology, Dissertations, No. 1108.

Hallgren, M., \& Olhager, J. (2006). Differentiating manufacturing focus. International Journal of Production Research, Vol. 44, N. 18-19, 3863-3878.

Hayes, R. H., \& Wheelwright, S. C. (1984). Restoring our competitive edge, competing through manufacturing. New York: John Wiley.

Interiano, J. R. (2004). Asociación Hondureña de Maquiladores. Recuperado el 18 de Julio de 2014, de Historia de la Maquila en Honduras: http://www.ahmhonduras.com/wp-content/uploads/2011/08/historia-maquila-honduras.pdf

Kathuria, R. (2000). Competitive priorities and managerial performance: a taxonomy of small manufacturers. Journal of Operations Management, Vol. 18, N. 6, 627-641.

Khanchanapong, T., Prajogo, D., Sohal, A. S., \& Cooper, B. K. (2014). The unique and complementary effects of manufacturing technologies and lean practices on manufacturing operational performance. International Journal of Production Economics, 191-203.

Kroes, J. R., \& Ghosh, S. (2010). Outsourcing congruence with competitive priorities: impact on supply chain and firm performance. Journal of Operation Management, Vol. 28, N. 2, 124-143.

Leong, G. K., Snyder, D. L., \& Ward, P. T. (1990). Research in the process and content of manufacturing strategy. Omega, Vol. 18, N. 2, 109-122.

Martinez, V., \& Bititci, U. S. (2006). Aligining value propositions in supply chains. International Journal of Value Chain Management, Vol. 1, N. 1, 6-18.

Nakane, J. (1986). Manufacturing futures survey in Japan: A comparative survey 19831986. Tokyo: Waseda University, System Science Institute.

Nambirajan, T., \& Prabhu, M. (2011). Competitiveness of manufacturing industries in Union Territory of Puducherry (India): A critical analysis. En P. D. Jawahar, Towards Managerial Excellence: Challenges and Choices. Tiruchirappalli: Mcmillan Publishers India.

Noble, M. A. (1995). Manufacturing strategy: testing the cumulative model in a multiple country context. Decision Sciences, Vol. 26, N. 5, 693-721.

Phusavat, K., \& Kanchana, R. (2007). Competitive priorities of manufacturing firms in Thailand. Industrial Management \& Data Systems, Vol. 107, N. 7, 979-996.

Porter, M. E. (1980). Competitive strategy: Techniques for analyzing industries and competitors. New York: The Free Press.

Porter, M. E. (1998). Competitive strategy: Techniques for analyzing industries and competitors. New York: The Free Press.

Rostek, K. (2012). The reference model of competitiveness factor for SME medical sector. Economic Modelling, Vol. 29, 2039 - 2048. 
Rudberg, M., \& Olhager, J. (2003). Manufacturing networks and supply chains: an operations strategy perspective. Omega, Vol. 31, N. 1, 29-39.

Sarache Castro, W. D., Castrillón, O. D., \& Giraldo, J. A. (2011). Prioridades competitivas para la industria de la confección. Estudio de caso. Cuadernos de Administración, Vol. 24, N. 43, 89-110.

Seguino, S., \& Grown, C. (2006). Gender equity and globalization: Macroeconomic policy for developing countries. Journal of International Development, Vol. 18, N. $8,1081-1104$.

Serna, H. (2007). Gerencia estratégica. Teoría-metodología, alineamiento, implementación y mapas estratégicos indices de gestión, 9 ed. Bogotá: 3R Editores.

Skinner, W. (1969). Manufacturing - Missing link in corporate strategy. Harvard Business Review, Mayo/Junio, 136-145.

Swink, M., \& Nair, A. (2007). Capturing the competitive advantages of AMT: Designmanufacturing integration as a complementary asset. Journal of Operations Management, Vol. 25, N. 3, 736-754.

Tan, K. C., Kannan, V. R., \& Narasimhan, R. (2007). The impact of operations capability on firm performance. International Journal of Production Research, Vol. 45, N. 21, $5135-5156$.

Terrádez, M. (22 de Agosto de 2014). Universitat Oberta de Catalunya. Obtenido de Análisis de Componentes Principales: http://www.uoc.edu/in3/emath/docs/Componentes_principales.pdf

Vivares-Vergara, J. A., Sarache-Castro, W. A., \& Narango-Valencia, J. C. (2014). The content of manufacturing strategy: A case study in Colombian industries. Dyna, Vol. 81, N. 183, 140-147.

Ward, P. T., McCreery, J. K., Ritzman, L. P., \& Sharma, D. (1998). Competitive priorities in operations management. Decision Science, 1035-1046.

Yang, C.-L., Lin, S.-P., Chan, Y.-H., \& Sheu, C. (2010). Mediated effect of environmental management on manufacturing competitiveness: An empirical study. International Journal of Production Economics, Vol. 123, N. 1, 210-220.

"LA REVISTA INNOVARE NO SE HACE RESPONSABLE EN NINGÚN CASO DE LOS CONTENIDOS, DATOS, CONCLUSIONES U OPINIONES VERTIDAS EN LOS ARTÍCULOS PUBLICADOS, SIENDO ESTA RESPOSABILIDAD EXCLUSIVA DEL (DE LOS) AUTOR (AUTORES)" 